\title{
DIGITAL IMAGING BASICS \\ Performance Tests and Quality Control of Cathode Ray Tube Displays
}

\author{
Hans Roehrig, Hartwig Blume, Ting-Lan Ji, and Mike Browne
}

\begin{abstract}
Spatial resolution, noise, characteristic curve, and absolute luminance are the essential parameters that describe physical image quality of a display. This paper presents simple procedures for assessing the performance of a cathode ray tube (CRT) in terms of these parameters as well as easy set up techniques. The procedures can be used in the environment where the CRT is used. The procedures are based on a digital representation of the Society of Motion Pictures and Television Engineers pattern plus a few simple other digital patterns. Additionally, measurement techniques are discussed for estimating brightness uniformity, veiling glare, and distortion. Apart from the absolute luminance, all performance features can be assessed with an uncalibrated photodetector and the eyes of a human observer. The measurement techniques especially enable the user to perform comparisons of different display systems. () 1990 by W.B. Saunders Company.
\end{abstract}

KEY WORDS: CRT performance, CRT testing, CRT set up. SMPTE patterns.

D IGITAL IMAGING procedures are finding increasing application in diagnostic radiology. As a result, cathode ray tube (CRT) displays have become an integral part of the diagnostic radiology department. Efforts are underway to investigate the suitability of high-resolution CRTs for diagnosis by the radiologist. Initial results appear to be encouraging. ${ }^{1-4}$

Efforts are also in progress to precisely characterize the physical performance of high-resolution CRTs in comparison to films generated by laser image printers with comparable addressable pixel matrices and hung in front of a

From the Department of Radiology, University of Arizona, Tucson; Philips Medical Systems North America, Inc, Shelton, CT, and the Department of Electrical and Computer Engineering, University of Arizona, Optical Sciences Center, Tucson.

Supported by National Institute of Health Contract 5ROI-CA49261-02, "Development and Characterization of a Viewing Console," and by the AT\&T Bell Laboratories and Philips Medical Systems, University of Arizona project No. 473410).

Address reprint requests to Hans Roehrig, Department of Radiology, University of Arizona, Tucson, AZ 85724.

(c) 1990 by W.B. Saunders Company.

0897-1889-90/0303-0010\$03.00/0 lightbox. ${ }^{557}$ Initial results show that CRTs fall short in key performance features such as (1) the maximum luminance, which is about a factor of 10 below that of a light box, (2) veiling glare (scatter in the glass faceplate) that reduces the modulation transfer, (3) the number of resolvable picture elements, which is smaller than the number of addressable locations on the CRT screen, (4) spatial noise (phosphor granularity) is limiting the low-contrast detectability, and (5) dynamic range.

While it is conceivable that CRTs with lower performance than films generated by laser printers and displayed on light boxes may be used for primary diagnosis tasks, it is necessary that the user know the physical performance of the employed CRT.

The characterization of a display is by no means trivial. First, there are no standards that define quantitatively displayed image quality. A variety of standards come to mind concerning the input video signal quality for CRTs such as RS-170, RS 330 and RS-343. ${ }^{8-10}$ However, these standards do not cover CRTs with addressable pixel matrices in excess of $1024 \times 1024$, nor do they cover scan rates in excess of 60 frames per second. None of these standards specifies output signal properties. Often, the analog input signal into high-resolution CRTs seems to conform to the provisions set by Standard RS-170: the peak-to-peak voltage of the composite video signal is $1 \mathrm{~V}$, with $0.707 \mathrm{~V}$ devoted to the actual video signal, and the remainder devoted to the synchronization pulses. The polarity is usually negative, meaning the highest brightness is achieved with a voltage of $0 \mathrm{~V}$. A voltage of $-0.707 \mathrm{~V}$ results in "black".

Secondly, the precise physical performance evaluation of a CRT requires instrumentation that is available in only a few laboratories. ${ }^{5}$ However, some easy ways exist to obtain semiquantitative information on the performance of a CRT and to assure proper and optimum operation for a given display task..$^{8-17}$ This communication reviews these tests and offers experimental 
results in comparison to tests performed with a sophisticated evaluation facility.

The proposed tests provide a rather complete image quality assessment and can be performed in the clinical environment. Only a few of the tests should be performed daily as part of a quality assurance program.

For a variety of performance features, quantitative results are not necessary. It is adequate to determine whether given input patterns can be perceived or suspected artifacts are present or absent to the human observer. The general philosophy should be that if the human observer cannot detect a deficiency, then the system settings should not be changed.

Recently the operation of CRTs has been described in detail in this journal. ${ }^{18}$ This article may serve as a background to the communication presented on the following pages.

\section{PERFORMANCE CHARACTERISTICS OF INTEREST AND THEIR DEFINITIONS}

Absolute luminance refers to the amount of visible light emitted in the center of the display surface and is expressed in units of foot-lambert (ft-L).

Dynamic range is defined by the ratio of maximum-to-minimum luminance of the ideal display function. As stated above, due to veiling glare, the actually realized display function and, for this reason, the maximum-to-minimum luminance ratio of a real image depends on the displayed scene. Correspondingly, a dynamic range figure may be derived from the step tablet of the Society of Motion Pictures and Television Engineers (SMPTE) pattern.

Characteristic curve or display function relates the CRT's luminance to the input signal. It is obtained by successively addressing the entire active display area with the same signal and measuring the luminance in the center. This display function is an ideal function which, in general, due to veiling glare, CRTs cannot maintain when displaying a nonuniform, grey scale image. An example of the actual grey scale reproduction in an image can be derived from the SMPTE pattern by measuring the luminance levels of its step tablet.

Luminance uniformity is characterized by a pseudo three-dimensional plot of the luminance versus the location of the active display area. In order to reflect the visual impression of the nonuniformity, the measured luminance is presented on a logarithmic scale.

Veiling glare is the internal scatter, caused primarily by light scatter within the CRT glass envelope. Veiling glare constitutes the shallow, very long tail of the point spread functions, resulting in a reduction especially of mid and high-frequency modulation transfer.

Noise is a measure of the luminance fluctuations when the command level is constant. CRTs produce temporal and spatial noise. Temporal noise is related to noise in the CRT's amplifying circuits, fluctuations in the intensity of the electron beam, instabilities in the voltage applied between the electron gun and the phosphor, and variations in the phosphor decay. Spatial noise is related to the phosphor grain structure and nonuniformities caused by the method of phosphor deposition. We are interested in temporal frequency components from a few tenths of $1 \mathrm{~Hz}$ to 6 to $8 \mathrm{~Hz}$, ie, in modulation periods longer than the integration time of the eye-brain, which, therefore, may affect detail perception. We assume that the CRT exhibits no perceivable flicker. In the spatial domain, we concentrate on the mid to high-frequency noise spectrum and will not consider large-scale luminance nonuniformities here. Noise is quantified by single-pixel signal-to-noise ratios.

Modulation transfer is defined here as the ratio of the just perceivable degree of modulation of a square wave pattern to the threshold modulation of human observers. A square wave response or contrast transfer function is obtained when plotting these ratios as a function of spatial frequency.

Often, the number of actually resolvable pixels is smaller than the number of addressable pixels. The former is determined by the size of the electron beam and the bandwidth of the display system. The diameter of the electron beam is a function of the electron beam current and typically a function of the location within the CRT's active area. The degree of input modulation required to resolve neighboring addressable pixels is affected by veiling glare.

Distortion is the amount of geometrical displacement of a pixel from its correct location. It is characterized by a two-dimensional map of the distorted grid with respect to the possibly trans- 
lated and rotated perfect grid. Distortion may be quantified by the maximum two-dimensional displacement referred to the diagonal of the CRT and expressed in percent.

\section{MATERIALS AND METHODS}

\section{Test Patterns}

SMPTE pattern. The SMPTE pattern" is a comprehensive and widely used test pattern. It can be used for set up, acceptance testing, and day-to-day operational checks and adjustments of focus, brightness and contrast settings as well as for assessing dynamic range, resolution, step response, display uniformity, and the display function. The pattern was developed in the early 1980s by members of a subcommittee of the SMPTE and with the cooperation of professional organizations from the medical field (clinicians as well as health physicists).

Typically, the SMPTE test pattern is generated by a computer program. It can be generated for square as well as for rectangular formats. Its data range is selected to match the digitization resolution of the video display buffer used $(8$ bits, 10 bits, etc.). Since the bit depth of video display buffers varies, signal levels are quoted in percent of full scale rather than in units of grey levels. Care has to be taken that the digital representation of the SMPTE pattern also accurately matches the pixel matrix of the particular CRT display with which it is to be used. The bar patterns for testing spatial resolution may otherwise have the wrong frequency.

Digital representations of the SMPTE pattern are normally delivered by the suppliers of the display systems as part of the display controller software package.

Figure $l$ is a reproduction of the SMPTE pattern. The particular features of the pattern and their purpose are: (1) a uniform background set at 50\% input level over a major portion of the pattern area. It is provided to test gross display nonuniformities, phosphor grain, phosphor burn, and the proper functioning of the raster display and erase circuits; (2) a rectangular white grid of at least 11 evenly spaced lines each in horizontal and vertical direction (100\% video signal) for the purpose of checking geometric distortion; (3) a grey step pattern of 11 steps of equally spaced input levels from $0 \%$ to $100 \%$ video signal for the purpose of measuring dynamic range and characteristic curve; (4) two small contrast steps, $5 \%$ in magnitude, (a) a smaller square set at $5 \%$ input level within a larger square at 0 input and (b) a smaller square set at $95 \%$ input level embedded in a larger square set at $100 \%$ input. This is perhaps the most important feature of the test pattern. The purpose is to permit fine tuning of the brightness contrast settings of the display and to assure that all shades of grey can be perceived in the image; (5) five high-contrast resolution patterns (one each in the four corners of the pattern and one in the center) of three spatial frequencies each for the purpose of testing spatial resolution and modula-

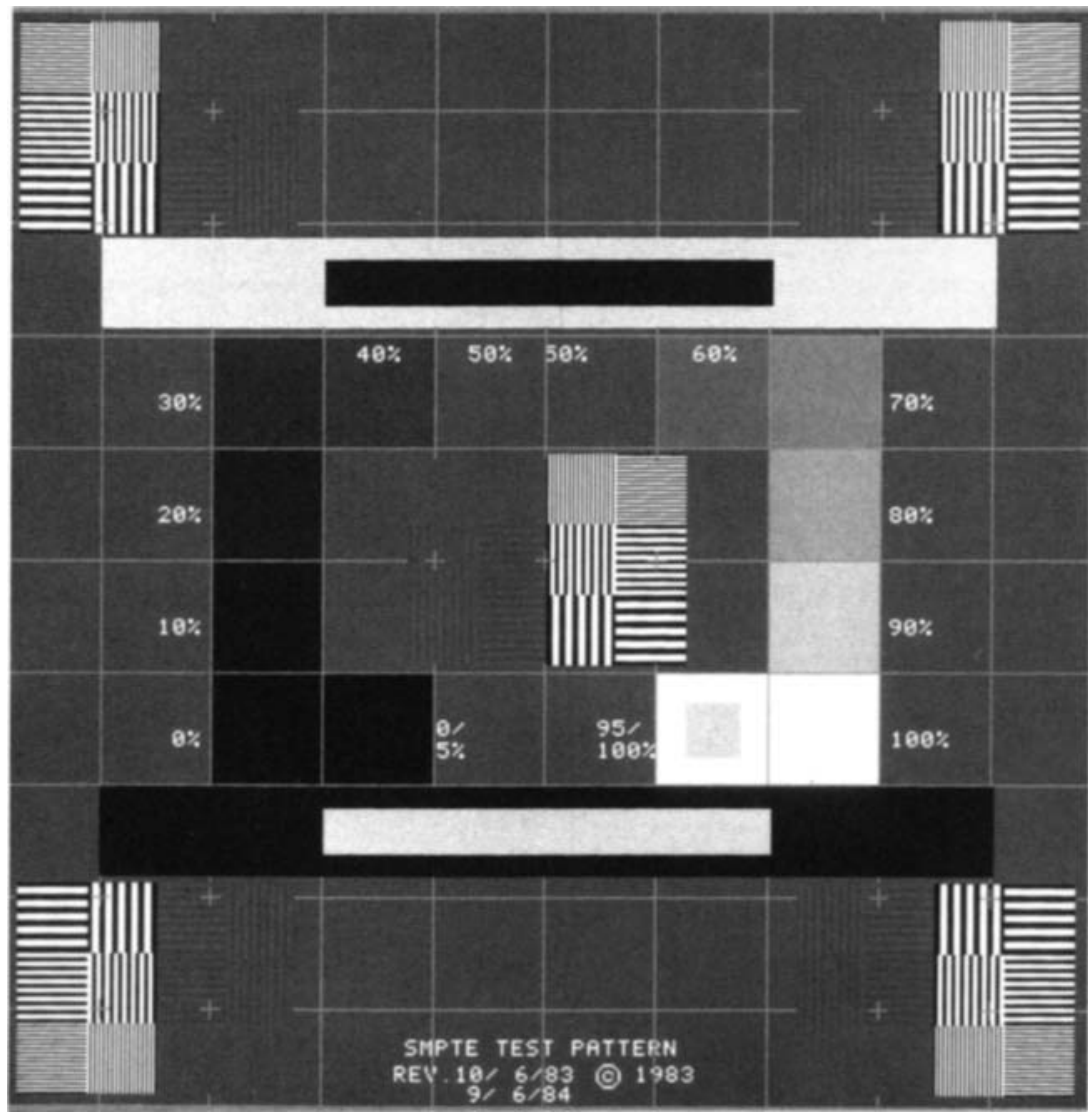

Fig 1. Photograph of SMPTE pattern. 
tion transfer. The resolution patterns are oriented both horizontally and vertically. The spatial frequencies are: $1,1 / 2$, and $1 / 3$ times the Nyquist frequency of the pixel matrix; (6) low-contrast resolution patterns of three different degrees of input modulations each, but with only one spatial frequency, next to each of the five high-resolution patterns, for the purpose of testing the effects of noise (spatial as well as temporal) and veiling glare. The amounts of modulation are $1 \%, 3 \%$, and $5 \%$; the spatial frequency is $1 / 2$ the Nyquist frequency of the pixel matrix; (7) a white window with black insert (in the upper half of the pattern) and a black window with white insert (in the lower half of the pattern) for the purpose of inspecting the step response of the display (midband streaking, low-frequency response, video amplifier ringing).

Test patterns generated at control console. Most CRTs in the radiology department are operated from a display buffer, located in the control console of an imaging system whose images are to be displayed by the CRT. The control console usually offers software to generate data that can be used to test individual performance features of the CRT. Test patterns generated by such software include:

Pattern 1 for assessing luminance uniformity and measuring the display function. Uniform grey fields cover the entire display screen at different input levels (Fig 2).

Pattern 2 estimates geometric distortion. It includes a rectangular grid of horizontal and vertical lines (Fig 3), and a high-density array of the letter $\mathbf{E}$. The letters have to be evenly spaced horizontally as well as vertically. The size of the letters should extend vertically over at least 12 lines and horizontally over at least 5 pixels (Fig 4).

Pattern 3 is for estimates of veiling glare (scatter). It uses a square of $200 \times 200$ pixels in the center of the CRT of zero input signal embedded in a uniform background set at $100 \%$ input signal (Fig 5).

Pattern 4 is used for assessing step response. In the upper half of the CRT's active area is a rectangular white window (200 pixels horizontally by 100 pixels vertically; $100 \%$ input signal) in a black background ( $0 \%$ input signal), and in the lower half of the CRT's active area is a rectangular black window ( 200 pixels horizontally by 100 pixels vertically; $0 \%$ input signal) in a white background (100\% input signal) (Fig 6).

Pattern 5 is used for estimating the magnitude of noise (temporal and fixed pattern noise). A detail-contrast pattern,

Signal level: $1 \%$ to $100 \%$

Fig 2. Digital pattern for testing luminance uniformity.

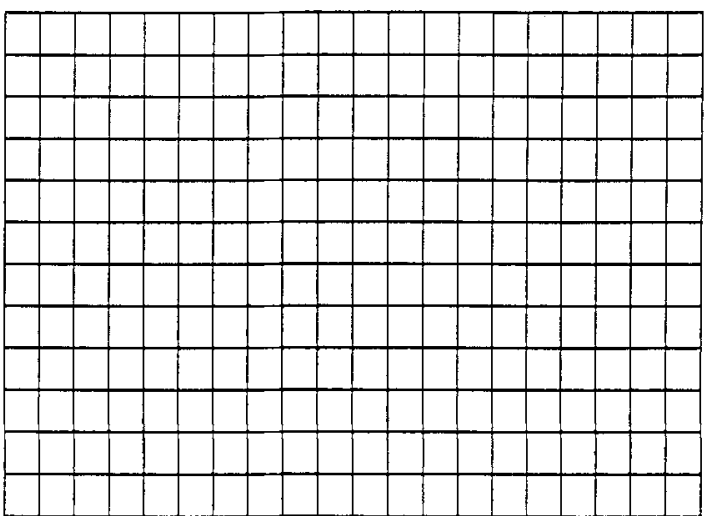

Fig 3. Digital pattern for testing geometrical display distortion.

consisting of an array of squares of increasing size from $1 \times 1$ pixel, $2 \times 2$ pixels, $3 \times 3$ pixels up to $25 \times 25$ pixels is embedded in a uniform background with provision to vary the input contrast (from $0.5 \%$ to $10 \%$ ) and to change the input level of the background (Fig 7).

Pattern 6 is used for estimating the contrast transfer function. It consists of an array of horizontal and vertical squarewave patterns of four high-spatial frequencies (in units of Nyquist frequencies $f_{N}: f_{N}, 1 / 2 f_{N}, 1 / 3 f_{N}$, and $1 / 4 f_{N}$ ), with provisions to vary the modulation from $0.4 \%$ to $3.9 \%$ (Fig 8). Each "patch" of a particular spatial frequency and orientation may be about $120 \times 120$ pixels in size. All squarewave patterns for one degree of modulation are displayed simultaneously.

\section{Instrumentation Including the Human Observer}

The selection of the equipment described below and used in experiments to demonstrate validity of the test procedures does not constitute an endorsement of the equipment by the authors.

Human observer. According to the philosophy expressed in the introduction, often qualitative and relative assessments of the display performance are adequate. In this sense, the human observer who, in the end, is the user of the display system is used as a measuring device for uniformity properties, resolution, and effects of noise on detail contrast per-

EEEEEEEEEEEEEEEEEEEEEEEEEEEEEEEEEE
EEEEEEEEEEEEEEEEEEEEEEEEEEEEEEEEEE
EEEEEEEEEEEEEEEEEEEEEEEEEEEEEEEEEE
EEEEEEEEEEEEEEEEEEEEEEEEEEEEEEEEEE
EEEEEEEEEEEEEEEEEEEEEEEEEEEEEEEEEE
EEEEEEEEEEEEEEEEEEEEEEEEEEEEEEEEEE
EEEEEEEEEEEEEEEEEEEEEEEEEEEEEEEEEE
EEEEEEEEEEEEEEEEEEEEEEEEEEEEEEEEEE
EEEEEEEEEEEEEEEEEEEEEEEEEEEEEEEEEE
EEEEEEEEEEEEEEEEEEEEEEEEEEEEEEEEEE
EEEEEEEEEEEEEEEEEEEEEEEEEEEEEEEEEE

Fig 4. Digital pattern for testing geometrical display distortion. 


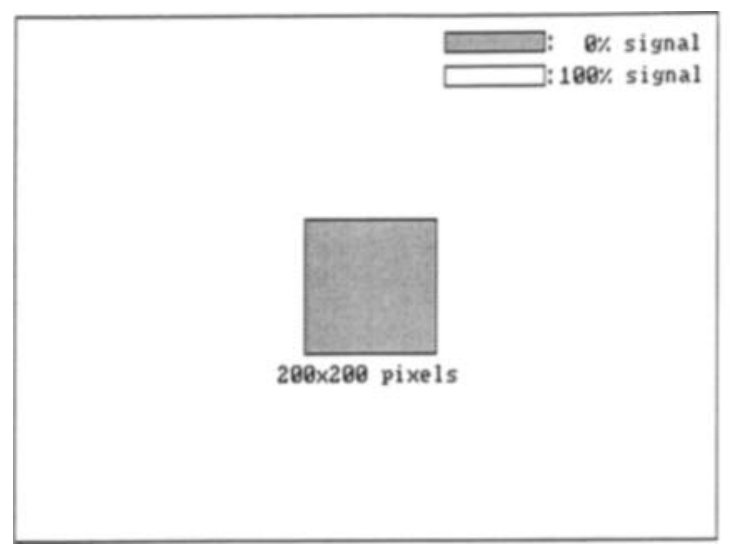

Fig 5. Digital pattern for estimating veiling glare.

ceptibility. Since the performance of the human observer regarding these features is relatively well known, some semiquantitative information can even be derived from the human measurements. Included here are typical human observer performance features concerning our tests.

The spatial frequency response of the human eye has the shape of a bandpass filter with a peak response at about 5 linepairs per degree (lp/deg) of viewing angle ${ }^{19}$ and decreasing sensitivity toward lower as well as higher spatial frequencies. The eye is very insensitive to gradual, large-area luminance nonuniformities. Typically, the human observer will have problems detecting a gradual luminance change of $20 \%$ to $30 \%$ across the active area of a CRT. ${ }^{19}$

The threshold modulation of human observers for detecting bar patterns is between $0.4 \%$ and $0.5 \%$ at spatial frequencies of about $3 \mathrm{lp} / \mathrm{deg}$ to $6 \mathrm{lp} / \mathrm{deg} .{ }^{19}$ This fact can be utilized to estimate the modulation transfer capabilities of a display system.

Threshold-signal-to-noise ratios governing the detection of aperiodic objects can be used to estimate the signal-to-noise ratio of a CRT. From the work of Rose ${ }^{20}$ and Rossell and Willson, ${ }^{21}$ it is known that a human observer can detect an object at a given object contrast relative to its background if the object contrast-to-noise ratio is above a certain threshold

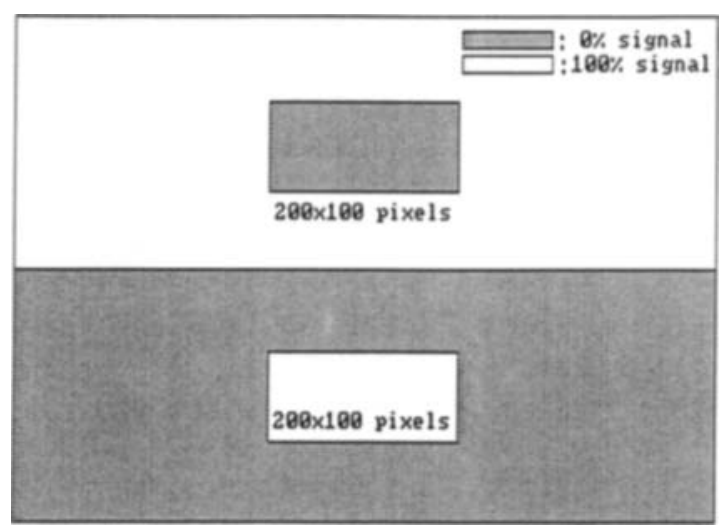

Fig 6. Digital pattern for assessing step response of the display system.

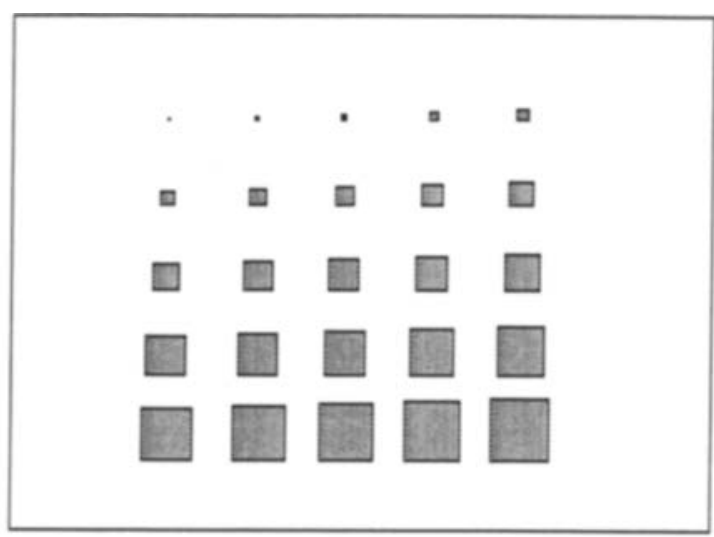

Fig 7. Digital pattern for determining contrast detail perception and effect of noise.

("display signal-to-noise ratio," Rose model ${ }^{20,21}$ ). The threshold signal-to-noise ratio for a probability of detection of $50 \%$ is about 3.

Contrast-detail performance of human observers with electronic imaging systems is comprehensively reviewed by Cohen. ${ }^{22}$

Magnifying glasses. Magnifying glasses with magnifications between 2 and 8 are used for the modulation measurements to bring the angular spatial frequencies of the various bar patterns from $3 \mathrm{lp} / \mathrm{deg}$ to $6 \mathrm{lp} / \mathrm{deg}$ for human observers.

Photodetector. Minimally, a relatively calibrated photodetector is needed for the performance assessment of the CRT display. Silicon photodiodes with a transimpedance amplifier are suitable for such assessments. Typically, the detector signal is displayed by a digital voltmeter. Photometric response and calibration are important only for the measurement of the CRTs luminance. But even here an uncalibrated detector will suffice if a luminance standard is available, such as the Model 220 from Gamma Scientific ${ }^{23}$ (San Diego, CA).

Photometric response can be achieved by placing a photometric filter like the one that comes with the EG\&G (San Diego, CA) Radiometer/Photometer $550^{24}$ over the detector. This filter matches the response of the detector to that of a standard human eye, ranging from about $410 \mathrm{~nm}$ to about $720 \mathrm{~nm}$ with a peak at $550 \mathrm{~nm}$. Photometric filters are not just green filters with maximum transmission at a wavelength of

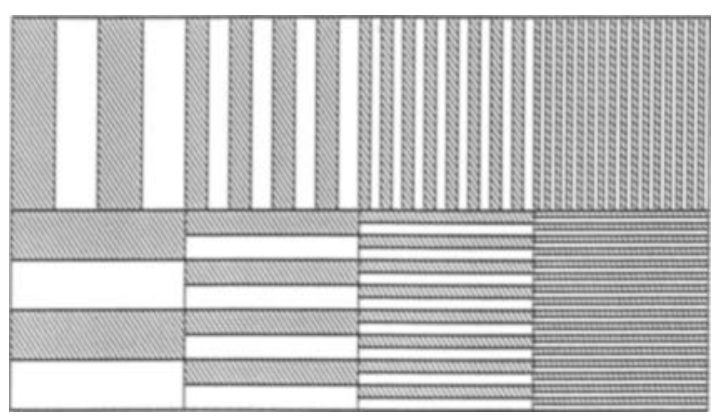

Fig 8. Digital pattern for estimating square wave response. 
$550 \mathrm{~nm}$ and cut off below $410 \mathrm{~nm}$ and above $720 \mathrm{~nm}$. Low transmission in the near infrared beyond $720 \mathrm{~nm}$ is particularly important if one wants to use a silicon detector, because many standard light sources emit a significant amount of power in the near infrared, and unfiltered silicon diodes exhibit good response in this spectral region.

Alternatively, one may select a silicon detector with a photometric response like the PIN-10 AP, available from United Detector Technology (Hawthorne, CA). ${ }^{25}$

An uncalibrated silicon detector, model UDT PIN-10 DP, (United Detector Technology, Hawthorne, CA) was used together with the transimpedance amplifier UDT-101C. ${ }^{25} \mathrm{~A}$ hand held three-digit digital multimeter, Fluke model 23, (J. Fluke Manufacturing Company, Inc, Everett, WA), was used to read the detector signals. The detector was placed at the entrance of a $15 \mathrm{~cm}$ long tube with about $4 \mathrm{~cm}$ ID. Two 7 $\mathrm{mm}$ diameter apertures, one located in the middle of the tube and one at its front end, served as baffles to restrict the field of view to $7 \mathrm{~mm}$. The photometric green filter could be placed inside the tube right in front of the detector.

Ideally, a lens should be used to image the CRT area of interest onto the photodetector. However, if the detector's active area is not larger than a few millimeters in diameter, and if the detector is located in a $15 \mathrm{~cm}$ long and about 5 $\mathrm{cm}$-wide tube with at least two baffles to restrict its field of view to a few degrees, the detector assembly can be used without a lens by placing it firmly against the CRT's glass faceplate. ${ }^{26}$ Its spatial resolution should be a few millimeters.

The detector and its amplifier must respond linearly over the wide luminance range of several orders of magnitude of the CRT. It is recommended to display the detector signal on an oscilloscope to check for detector saturation.

Caution is recommended with digital meters for readout. Digital meters sample the signal at a particular sampling rate, and this sampling rate is most likely different from the CRT's frame rate. Accordingly, beat frequencies may be generated, given by the sum and the difference between the sampling rate and the CRT's frame rate. As a result, the meter reading will vary from a maximum to a minimum value. The correct reading is the average over the maximum and minimum values.

Luminance standard. A luminance standard will be necessary for absolute luminance measurements, if a calibrated detector with a photometric response is not available. We have mostly used a Gamma Scientific calibrated source, Model $220,{ }^{23}$ with a nominal luminance of $100 \mathrm{ft}-\mathrm{L}$. Using the EG\&G Radiometer/Photometer, Model 550, (EG\&G ElectroOptics Division, San Diego, CA) its luminance was recalibrated and found to have a luminance of $124 \mathrm{ft}-\mathrm{L}$. Unfortunately, Model 220 is no longer on the market. An alternative is the Gamma Scientific calibration light source RS-13 ${ }^{27}$ (Gamma Scientific, Inc, San Diego, CA).

\section{CRT Set Up}

The set up procedures discussed in this section are designed to assure proper operation of the CRT, before the tests are performed. These set up procedures, however, should also be part of a daily quality assurance program. The time involved for a daily quality assurance program is minimal: the SMPTE pattern is displayed and inspected. Action is only necessary if the image of the SMPTE pattern does not meet specifications.

Brightness-contrast controls. Almost every CRT has analog controls marked "brightness" and "contrast." The brightness control adjusts the beam current and represents means to provide a brightness "offset." The contrast control adjusts the gain of the video amplifier of the CRT.

The brightness-contrast controls basically need to be adjusted once for optimum presentation of grey scale to a human observer. To do this, the SMPTE pattern (with its step tablet) is displayed. The contrast-brightness controls are adjusted until both $5 \%$ contrast steps are well visible. Notice that the adjustment has to be done for the specific environment (ie, room lights) in which the CRT is to be used.

After these adjustments, the controls should be locked. From now on, any contrast enhancement or change in brightness should be done digitally by differently mapping the image data to the characteristic curve of the display, eg, by means of the precise and repeatable window and level settings of the display controller.

Focus adjust. In addition to the contrast-brightness controls, there usually also is a less accessible focus adjustment. The focus is optimally adjusted while a resolution pattern (SMPTE pattern or Pattern 6) is displayed. In general, high-luminance (corresponding to large beam current) means lower modulation transfer. However, this is not necessarily true. The operator should check resolution also while adjusting the contrast-brightness controls.

Warm up. The CRT should be turned on for warm up one hour before the intended test. Display systems often need time for the luminance output to stabilize.

Termination and T-connectors. High-resolution CRT displays operate at frequencies of more than $100 \mathrm{MHz}$ and digitization resolutions of at least 8 bits/pixel. Signals with such high bandwidth and precision need to be transmitted by coaxial cables of proper impedance and proper termination at the end of the cable. The output of most video sources, like display buffers, is terminated with 75 (sometimes 50) ohms. Therefore, one needs to use $75 \mathrm{ohm}$ cables (for example, RG-59, RG-179, or RG-11) or sometimes 50 ohm cables (for example RG 58 or RG 217) to connect the video signals to the CRT. Failure to terminate the cables properly leads to reflections that cause ghost images on the CRT.

T-connectors are often used for easy connection of other equipment (test equipment like oscilloscopes, for instance). Inadvertently, this equipment can disturb the proper termination or load down the video signal. Furthermore, T-connectors are often subjected to mechanical strain that may result in poor contact. Hence, care must be taken when using these connectors.

\section{TEST PROCEDURES}

\section{Quantitative Tests with the Photodetector}

Maximum absolute luminance. Display a uniform field with maximum input signal (100\%). Place the detector with the photometric filter into the center of the CRT's active area and record the detector signal $S_{\mathrm{CRT}}$. Then place the detector filter module against the luminance standard, the luminance of which is $\mathrm{L}_{\mathrm{st}}$. The detector signal is now $\mathrm{S}_{\mathrm{st}}$. The luminance 
of the CRT $\mathrm{L}_{\mathrm{CRT}}$ is found according to

$$
\mathrm{L}_{\mathrm{CRT}}=\mathrm{L}_{\mathrm{st}}\left(\mathrm{s}_{\mathrm{CRT}} / \mathrm{s}_{\mathrm{st}}\right)
$$

Dynamic range. Display first a uniform field with maximum input level (100\% signal) and afterward a uniform field with minimum input ( $0 \%$ signal). For both cases, place the detector into the center of the CRTs active area and record the detector signals $\mathrm{s}_{100 \%}$ and $\mathrm{s}_{0 \%}$. The maximum dynamic range $\mathrm{DR}_{\max }$ is found according to

$$
\mathrm{DR}_{\max }=\mathrm{s}_{100 \%} / \mathrm{s}_{0 \%}
$$

Correspondingly, display the SMPTE pattern and determine the ratio of the signal obtained in the $100 \%$ step versus the signal obtained in the $0 \%$ step:

$$
\mathrm{DR}_{\text {SMPTE }}=\left(\mathrm{s}_{100 \%, \mathrm{SMPTE}} / \mathrm{s}_{0 \%, \mathrm{SMPTE}}\right)
$$

The dynamic range within the SMPTE pattern is representative of the scatter generated by the particular luminance distribution in the SMPTE pattern. Maximum and SMPTE dynamic range will most likely be different. However, each one should be repeatable.

Characteristic curve. The characteristic curve of the CRT is obtained by measuring the luminance as a function of input level, ie, the ideal curve, by successively setting the entire display screen at one grey level; the grey scale reproduction within the SMPTE pattern is derived from the luminance levels of the step wedge within the pattern.

Luminance uniformity. Brightness uniformity is best determined by displaying a uniform field (say at $10 \%$ input level) (Fig 2). If luminance nonuniformities are visible, the relative luminance of at least 35 equally spaced locations of the CRT's active area is measured by holding the detector firmly against the glass faceplate of the CRT. Positional accuracy and repeatability is easily achieved by attaching to the CRT faceplate a black mask of the size of the CRT's active area with holes drilled at the 35 locations of interest. The diameter of the holes is chosen to match the diameter of the detector. Black, thin cardboard or 14 in $\times 17$ in $x$-ray film with uniform density of at least 2.0 are excellent materials for the mask.

Veiling glare. Display the following sequence of patterns: (1) a uniform field with maximum brightness (100\% input signal), (2) Pattern 3, the black square, and (3) a uniform field of minimum brightness ( $0 \%$ input signal). Place the detector into the center of the CRT's active area, (which should be the location of the center of the black square) and record the detector signals $\mathrm{s}_{\mathrm{i}}$ obtained for the three cases, $\mathrm{s}_{1}$, $s_{2}$, and $s_{3}$. A measure of the veiling glare (scatter) is found from

$$
V G=\left[\left(s_{2}-s_{3}\right) /\left(s_{1}-s_{3}\right)\right] 100 \%
$$

\section{Quantitative Tests Involving the Human Observer}

Spatial and temporal noise. Display the contrast detail pattern (Pattern 4, Fig 7) to several observers. For each observer, start with a high contrast value of $10 \%$ at a background signal of $100 \%$. Ask the observers to record the size of the object they can just barely detect. Then decrease the contrast slightly and again record the size of the object that the observer just barely discerns. As the contrast is decreased, the size of the smallest object that the human observer can just detect increases. According to the Rose model, the relation between the area $a_{0}$ of the object just noticed, the contrast $C$ with respect to the background, and the maximum displayed detail-signal-to-noise ratio per pixel $\mathrm{S} / \mathrm{N}$ is given by the following equation:

$$
a_{0}=\left[k^{2}(2-C)\right] /\left[(S / N)^{2} C^{2}\right]
$$

Here $\mathbf{k}$ is the threshold signal-to-noise ratio. This equation is solved to find $S / N$ to find the value of $S / N$ :

$$
\mathrm{S} / \mathrm{N}=\left[\mathrm{k}(2-\mathrm{C})^{1 / 2}\right] /\left[\mathrm{C}\left(\mathrm{a}_{0}\right)^{1 / 2}\right]
$$

Notice that this approach to measuring noise with the aid of the human observer constitutes the application of the Rose model to other than photon noise.

Squarewave response function. Display the array of horizontal and vertical squarewave patterns (Fig 8) for different degrees of modulation successively to four human observers. Ask them to find for each of the four spatial frequencies $f_{i}$ that modulation $m_{i}$ at which the presence of the pattern can barely be discerned. Ask them to use the magnifying glasses or adjust their viewing distances such that the various patterns appear to the observers under the optimum angular frequency between 3 and $6 \mathrm{lp} / \mathrm{deg}$ of viewing angle. Average the recorded results over the four observers to find $\mathrm{m}_{\mathrm{i}, \text { ave }}$ (expressed in percent).

For each of the spatial frequencies calculate the squarewave response factor $\mathrm{CTF}_{\mathrm{fi}}$ according to

$$
\mathrm{CTF}=\mathrm{m}_{\mathrm{t}} / \mathrm{m}_{\mathrm{i}, \text { ave }}
$$

Here $m_{t}$ is the threshold modulation of the human observer that is assumed to be constant. For a uniform, noiseless background, this value is mainly determined by the internal noise of the human observer, however, it may also be influenced by the amount of noise in the image. ${ }^{19}$ Furthermore, the threshold modulation may vary from observer to observer. Typically, $\mathrm{m}_{\mathrm{t}}=0.5 \%$. If the specific value of the threshold modulation for the specific observers is known and it is different from $0.5 \%$, then the respective value should be used.

The procedure can be modified by displaying the squarewave pattern with the provision to vary the modulation. This procedure will take longer, but it will be more accurate. Furthermore, since the squarewave patches are relatively small compared to the active area of the CRT screen, the pattern may be displayed at different locations on the CRT screen to test the uniformity of modulation transfer.

\section{Qualitative Tests Involving a Human Observer}

Distortion. For a semiquantitative analysis of distortion (scan linearity), display Pattern 2 (Fig 3). View the grid from a distance of at least $1 \mathrm{~m}$. Check both for systematic and for random deviations of the displayed grid from a perfect grid. Pincushion distortion, barrel distortion, and rotation of the grid pattern are defects that are particularly easy to spot.

An even more qualitative method, although fairly sensitive with respect to localized, random distortions, is the display of the array of capital Es (Fig 4). Here, the observer views the CRT and may record the location of the distortion.

Step response. The SMPTE pattern or Pattern 4 with a white window in a black background similar to the one shown in Fig 6 is displayed on the CRT. Insufficient electrical 
bandwidth or nonconstant response within the passband will lead to the appearance of a horizontal grey shadow in the white window to the right of the transition from black to white. Similarly, there also may be a horizontal grey shadow in the black background to the right of the transition from white to black at the right edge of the white window. Correspondingly, there may be streaks to the right at the beginning of the white window and to the right of the end of the white window.

Notice that these streaks appear in the horizontal direction, decreasing in intensity from left to right. Obviously, ideally the rise time of the system should be smaller than the beam dwell time (or, equivalently, the diameter) of a pixel to yield perfect step response.

\section{RESULTS}

The following results of tests performed on a high-resolution CRT, which had been evaluated with the equipment described in references 5,6 and 7 , demonstrate the validity of the simplified performance assessment procedures.

\section{Maximum Absolute Luminance}

Using the photodetector with the green photometric filter and an amplifier gain of $10^{8}$, the detector's reading was $0.916 \mathrm{~V}$ when held against the standard. When held against the CRT that was set at $100 \%$ input signal, the detector's reading was $0.317 \mathrm{~V}$ at the same amplifier gain of $10^{8}$. We estimate a CRT luminance of $42.9 \mathrm{ft}-\mathrm{L}$. With the EG\&G Radiometer/Photometer model 550 , the CRT's luminance is measured to be 39.5 $\mathrm{ft}-\mathrm{L}$. The error is only $8.6 \%$.

\section{Display Function and Dynamic Range}

The ideal display function (characteristic curve) was measured with an uncalibrated photo-

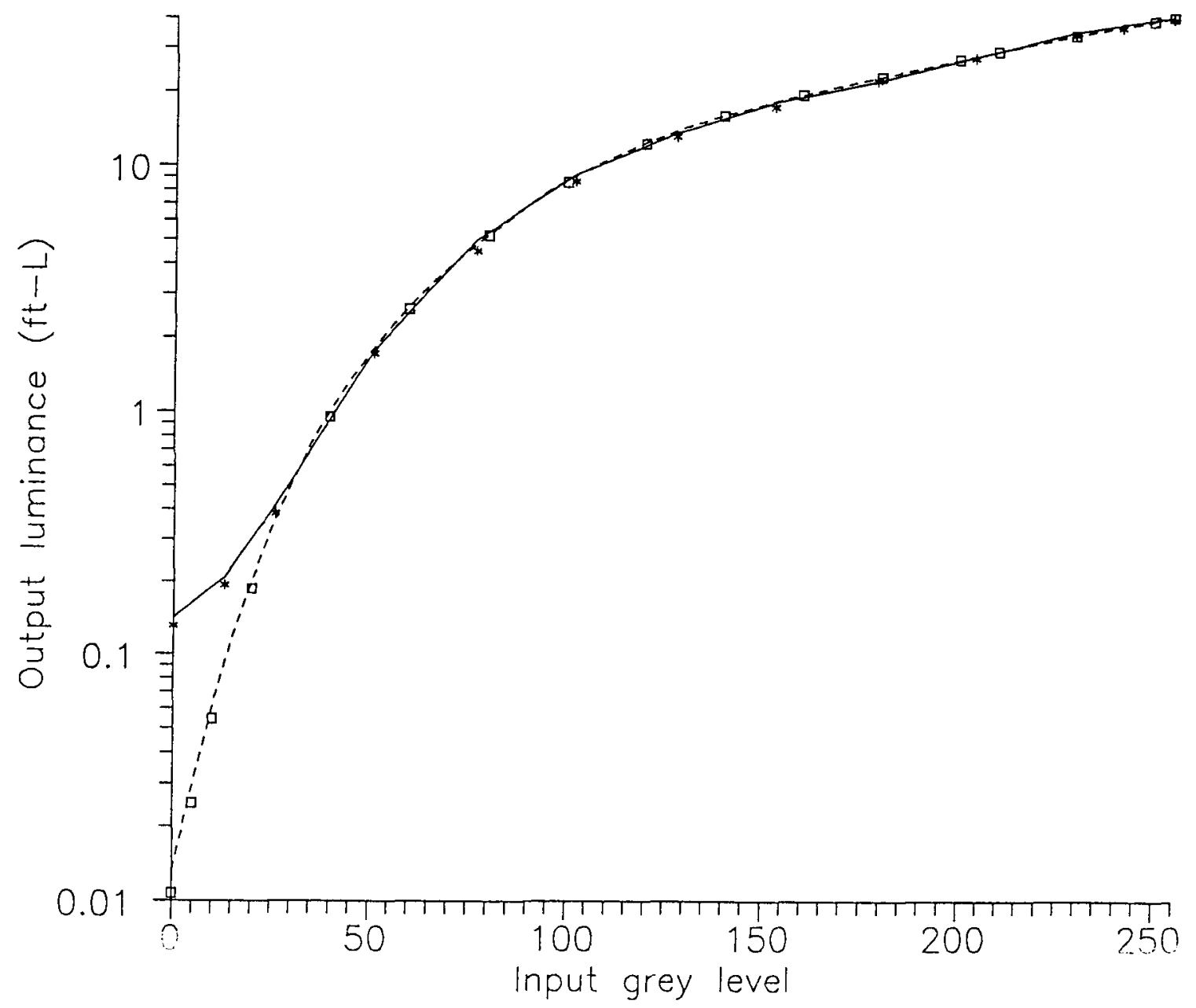

Fig 9. Characteristic curve and reproductions of grey scale in SMPTE pattern of a high-resolution CRT display system measured with different photodetectors. The curve (PMT) was obtsined from measurements on the test facility described in reference 5. 


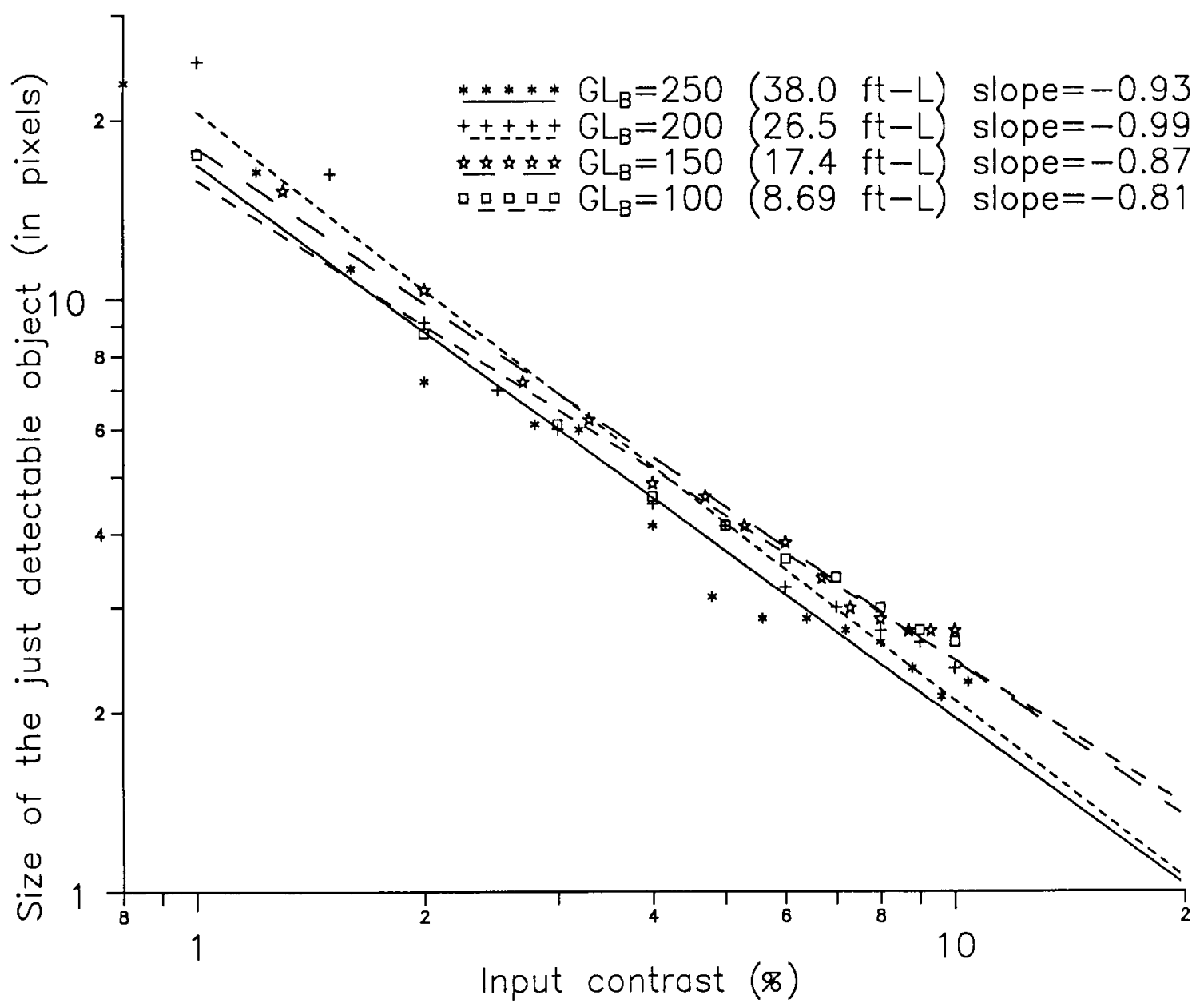

Fig 10. Detail contrast perceptibility. Just perceivable object size as a function of contrast with input level as parameter of high-resolution CRT display system.

detector and is plotted in Fig 9, using squares as symbols. From the data, a maximum dynamic range of $\mathrm{DR}_{\max }=3680$ was derived. The dotted curve in figure 9 shows in the dotted curve the display function obtained with our precision photometer/high-resolution analog-to-digital converter combination. Here a maximum dynamic range of $D R_{\max }=2977$ was obtained.

Figure 9 also shows the grey scale representation in the SMPTE pattern. The data plotted using stars as symbols were obtained by placing the detector/tube with baffles, but without the photometric green filter, into the center of each of the 11 steps of the grey step tablet. Finally, Fig 9 shows in the solid curve the actual grey scale reproduction that had been measured previously by sampling the luminance of the 11 steps of the SMPTE pattern with the aid of a photomultiplier/analyzing-aperture/lens combination. Notice again how close the curves are.
The dynamic range within the SMPTE pattern as estimated with the aid of the hand held silicon detector was $\mathrm{DR}_{\text {SMPTE }}=288$. This value is close to the value of $D_{\text {SMPTE }}=276$ estimated from the data obtained with the photomultiplier.

\section{Veiling Glare}

We displayed three patterns in the following order: (1) a uniform field with maximum brightness (100\% input signal), (2) Pattern 3 (Fig 5), and (3) a uniform field of minimum brightness ( $0 \%$ input signal). The three resulting signals were $\mathrm{s}_{1}=1.33 \mathrm{~V}, \mathrm{~s}_{2}=0.0184 \mathrm{~V}$, and $\mathrm{s}_{3}=$ $0.00033 \mathrm{~V}$ (using an amplifier gain of $10^{8}$ ). Using equation 4 , we find for the veiling glare $\mathrm{VG}_{2}=$ $1.36 \%$ or, for this luminance distribution, a maximum large-area contrast ratio (local dynamic range) of 73.5 .

We also evaluated the veiling glare by using the SMPTE pattern and a uniform black field. 


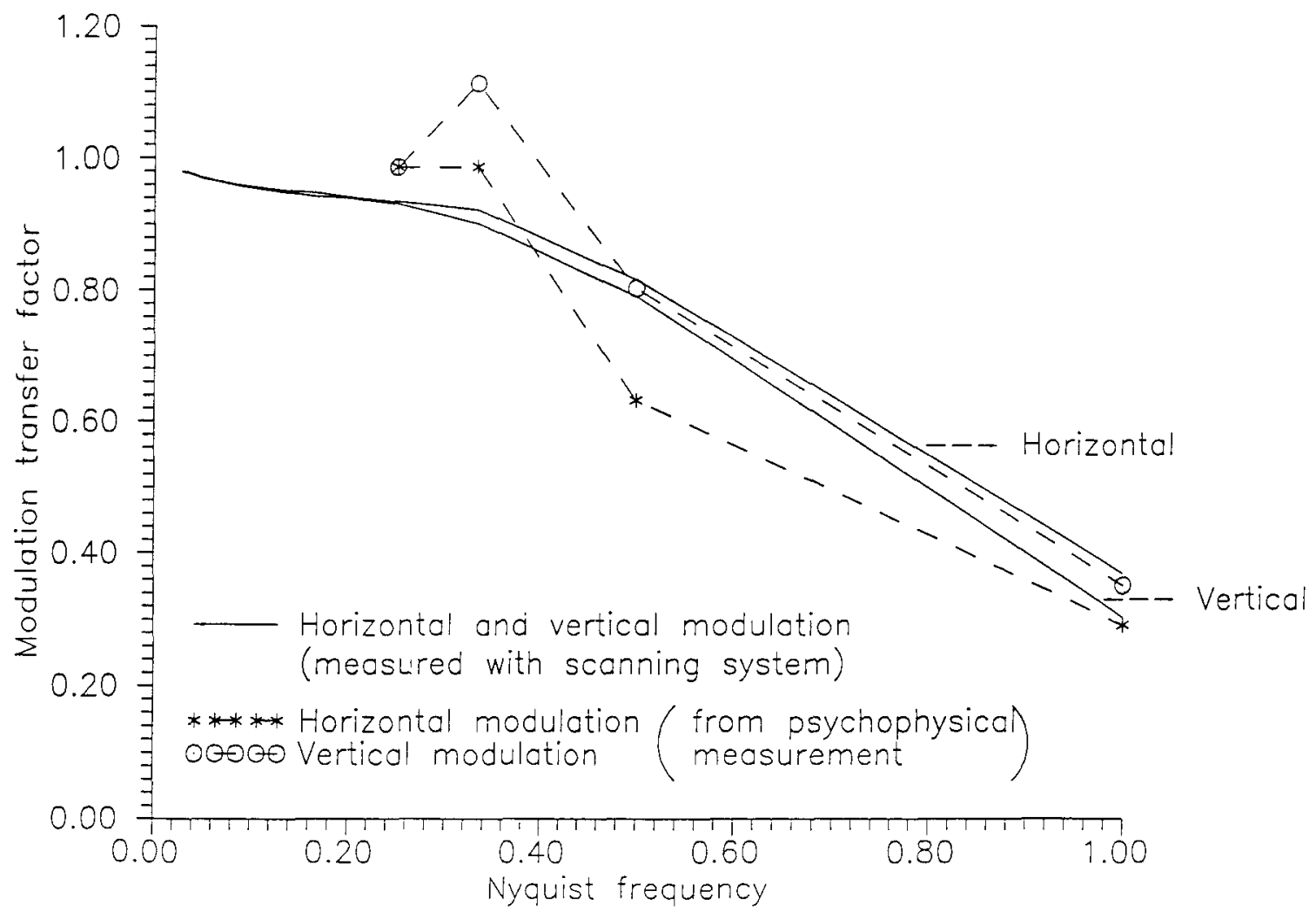

Fig 11. Square wave response in horizontal and vertical direction of a high-resolution CRT display system. The solid lines represent data measured with the test facility of reference 5.

The detector was placed into the $100 \%$ field, the $0 \%$ field, and finally at the center of the uniform black field. The corresponding signals were $\mathrm{s}_{1}=$ $1.29 \mathrm{~V}, \mathrm{~s}_{2}=0.0045 \mathrm{~V}$, and $\mathrm{s}_{3}=0.00033 \mathrm{~V}$ (again with a gain of $10^{8}$ ). Using equation 4 we find a local veiling glare $V G 1=0.35 \%$ or a local large-area contrast ratio (local dynamic range) of 288 .

Notice, in the center of Pattern 3, veiling glare is almost a factor 4 larger than in the center of the zero-input step of the SMPTE pattern. This illustrates how strongly the magnitude of veiling glare depends on the distribution and size of highlight areas in an image on the CRT.

\section{Signal-to-Noise Ratio}

We displayed the contrast detail pattern with various fixed contrasts to four observers. The contrast ranged from $1 \%$ to $10 \%$. Four background values were chosen: $33.8 \mathrm{ft}-\mathrm{L}, 24.0 \mathrm{ft}-\mathrm{L}$, $15.6 \mathrm{ft}-\mathrm{L}$, and $7.4 \mathrm{ft}-\mathrm{L}$.

The results are shown in Fig 10. The linear dimension of the just noticeable square (in units of pixel number) is plotted on double logarithmic scales as a function of the contrast with the luminance as parameter.

Notice first that the slope of the lines is about -0.9 that is very close to the ideal value of -1.0 (equation 6). Furthermore, the lines for the four different luminance levels are very close to each other, indicating independence of the limiting noise on the background brightness.

Using a $\mathrm{k}$ value of 2.9 in equation 7 , we find a value of 21 for the display signal-to-noise ratio per pixel. This value of 21 is very close to the value of 22 of the spatial signal-to-noise ratio which had been obtained from scanning a slit across a portion of the same CRT's active area. ${ }^{7}$ The spatial signal-to-noise ratio is, for all practical purposes, independent of the CRT luminance.

\section{Squarewave Response Function}

An array of horizontal and vertical squarewave patterns of four spatial frequencies (in units of Nyquist frequency $f_{N}: f_{N}, 1 / 2 f_{N}, 1 / 3 f_{N}$, and $1 / 4$ 
$\mathrm{f}_{\mathrm{N}}$ ) was displayed to five observers. In addition to the spatial frequency, the modulation was a variable: the pattern included the following 11 degrees of modulation $(0.2 \%, 0.4 \%, 0.6 \%, 0.8 \%$, $1.0 \%, 1.4 \%, 1.8 \%, 2.2 \%, 2.6 \%, 2.9 \%$, and $3.9 \%$ ). All in all, there were a total of 72 different squarewave patterns simultaneously displayed on the CRT.

The observers recorded the degree of modulation at which they could just discern the various bar patterns. The results were averaged over the five observers, and then the squarewave response (contrast transfer) factors were calculated, assuming that all observers had the same threshold modulation. We applied a threshold modulation of $\mathrm{m}_{\mathrm{t}}=0.433 \%$. This value was obtained in a previous experiment that estimated the degree of displayed modulation on the CRT for the input modulation at which the observers just barely saw the particular patterns, making use of the measured output modulations for $100 \%$ input modulation.

The calculated modulations are plotted in Fig
11 as a function of the spatial frequency. Fig 11 also shows the modulations, measured on the same high-resolution CRT with the aid of our scanning equipment. Notice that the general trend is well preserved. The individual values of modulation are different from the measured ones by up to about $30 \%$.

\section{CONCLUSION}

Knowledge about the physical performance of CRT displays is essential to the proper application of these display systems. Test patterns and test methods are available to permit quick set up and performance tests of CRTs in the environment of their operation. The test patterns are digitally generated by the host computer of the display system and inspected visually or by a relatively inexpensive photodetector. Even though the tests are simple and largely semiquantitative, they provide the necessary information accurately as is established by comparison with scientific measurement techniques.

\section{REFERENCES}

1. Cox GG, Cook LT, McMillan JH, et al: High resolution $2560 \times 2048 \times 12$ bit digital displays for chest radiography-A comparison with conventional film and digital hardcopy. Radiological Society of North America 1989 Scientific Exhibit No. 507

2. Frank MS, Jost RG, Blaine GJ, et al: Interpretation of mobile chest radiographs from a high resolution CRT display. Radiology 173P:401, 1989

3. Widoff B, Aberle DR, Brown K, et al: Hard copy versus soft copy display of 2000 digital chest images: ROC study with simulated lung nodules. Radiology 173P:401, 1989

4. Hayrapetian A, Aberle DR, Huang HK, et al: Comparison of 2048-line digital display formats and conventional radiographs: An ROC study. Am J Radiol 152:1113-1118, 1989

5. Roehrig H, Dallas W, Ji TL, et al: Physical evaluation of CRTs for use in digital radiography. Proc SPIE 1091:262278,1989

6. Blume $\mathrm{H}$, Roehrig $\mathrm{H}$, Browne $\mathrm{M}$, et al: Comparison of the physical performance of high resolution CRT displays and films recorded by laser image printers and displayed on light-boxes and the need for a display standard. SPIE 1232:97-114, 1990

7. Roehrig H, Ji TL, Browne M, et al: Signal-to-noise ratio and maximum information content of images displayed by a CRT. SPIE 1232:115-133, 1990

8. Gray JE, Stears J, Wondrow M: Quality control of video components and display devices. Proc International Course and Symposium on Electronic Imaging in Medicine, March, 1983
9. Harshbarger JH: Video standards and performance tests of video systems. Proc SPIE 486:72-76, 1984

10. Harshbarger $\mathbf{J H}$ : Standardization of television raster for nonbroadcast applications. J SMPTE 91:797-807, 1982

11. Lisk KG: SMPTE test pattern for certification of medical diagnostic display devices. Proc SPIE 486:79-82, 1984

12. Gray JE, Lisk KG, Haddick DH, et al: Test pattern for video displays and hard-copy cameras. Radiology 154:519527, 1985

13. Vii Model 110M Medical Imaging Video: A video standard for medical imaging equipment of all types. Product of Visual Information Institute, Inc, Xenia, OH, 45385-0033

14. Gray JE, Stears J, Wondrow M: Quality control of video components and display devices. Proc SPIE 486:64-71, 1984

15. Visual Information Institute, Inc: Display linearity evaluation. Publication Number 011-1083, Xenia, OH, 453850033,1983

16. Visual Information Institute, Inc: Recommended practice: Performance test for medical imaging monochrome display devices. Publication Number 013-0185, Xenia, $\mathrm{OH}$ 45385-0033, 1987

17. Visual Information Institute, Inc: Recommended practice: Performance test for video display devices. Publication Number 009-0983, Xenia, OH 45385-0033, 1984

18. Keller PA: Cathode-ray tube displays for medical imaging. J Dig Imag 3:15-25, 1990

19. Pollehn H, Roehrig H: Effect of noise on the modulation transfer function of the visual channel. J Optical Society of America 60:842-848, 1970 
20. Rose A: Vision: Human and Electronic. New York, NY, Plenum, 1973

21. Rosell F, Willson HL: Recent psychophysical experiments and the display signal-to-noise ratio concept, in Biberman LM (ed): Perception of Displayed Information, New York, NY, Plenum, 1971 pp 167-232

22. Cohen $\mathrm{G}$ : Contrast-detail analysis of imaging systems: Caveats and kudos, in Doi K, Lanzl L, Lin PJP (ed): Recent Developments in Digital Imaging, Med Phys Monograph No. 12, American Institute of Physics, New York, NY, 1985
23. Gamma Scientific Inc: 1977 Selection Guide for Light Measurement Systems. San Diego, CA, 1977

24. EG\&G Electro-Optics Division: Data Sheet L2011C1-1, 03/78. San Diego, CA, 1978

25. United Detector Technology: Silicon Photodetectors, Domestic Price List 9D1081. Hawthorne, CA, 1981

26. Smith WJ; Modern Optical Engineering. McGrawHill, New York, NY, 1966

27. Gamma Scientific Inc: RS Series Calibration Light Sources, Data Sheet No. 501, 11/80 5MC 